\title{
FOLIA
}

Amazónica

Revista del Instituto de Investigaciones

de la Amazonía Peruana

\section{CARACTERIZACIÓN ANATÓMICA Y FÍSICO MECÁNICA DE LAS FIBRAS DE “TAMSHI” (Heteropsis flexuosa (Kunth) G.S. Bunting) PROVENIENTE DE ECHARATE, LA CONVENCIÓN, CUSCO}

\author{
Enrique OLEA ${ }^{1}$, Héctor GONZALES ${ }^{2}$, Manuel CHAVESTA³ \\ Moisés ACEVEDO ${ }^{4}$, Juan BALUARTE ${ }^{5}$
}

1. Bach. en Ingeniería Forestal. Tesista de la Facultad de Ciencias Forestales. Facultad de Ciencias Forestales. Universidad Nacional Agraria La Molina. enrique.olea85@gmail.com

2. PhD., Ing. Forestal. Universidad Nacional Agraria La Molina. egonzales@lamolina.edu.pe

3. Ms. Sc., Ing. Forestal. Profesor principal de anatomía de la madera. Facultad de Ciencias Forestales. Universidad Nacional Agraria La Molina. mchavesta@lamolina.edu.pe

4. Ms. Sc., Ing. Forestal. Profesor principal de propiedades físicas y mecánicas de la madera. Facultad de Ciencias Forestales. Universidad Nacional Agraria La Molina. macevedo@lamolina.edu.pe

5. Dr., Ingeniero Forestal. Servicio Nacional Forestal y de Fauna Silvestre. jbaluarte@serfor.gob.pe

\section{RESUMEN}

La presente investigación tiene como propósito caracterizar la estructura anatómica y las propiedades físico-mecánicas de las raíces aéreas de Heteropsis flexuosa "alambre tamshi" (Araceae) expuestas a condiciones diferentes de humedad y tratamientos de envejecimiento. Las características de los elementos anatómicos de las raíces confirma la presencia de tres zonas visiblemente diferenciadas, observándose en la estela no periférica un gran número de fibras comprimidas que le proporcionan resistencia a las raíces. Respecto a los ensayos físicos, el contenido de humedad de las raíces en condición verde fue de 84,88\% y en condición seca al aire fue de 15,9\%; la densidad lineal fue 65,09 g/m. Los valores de las propiedades mecánicas de las raíces sin envejecimiento resultaron menores que aquellas obtenidas con envejecimiento; el alargamiento fue de $1,4 \%$ y $0,97 \%$ respecto a la longitud inicial, la TEA (tensil energy absorption) fue de $31138,07 \mathrm{~J} / \mathrm{m}^{2}$ y $15577,47 \mathrm{~J} / \mathrm{m}^{2}$, y la resistencia a la torsión de 1,065 $\mathrm{kg} / \mathrm{m}$ y $0,945 \mathrm{~kg} / \mathrm{m}$, respectivamente. Las raíces con envejecimiento mostraron 
comportamiento a la tracción de $584,78 \mathrm{Kg} / \mathrm{cm}^{2}$, y sin envejecimiento de 535,92 $\mathrm{Kg} / \mathrm{cm}^{2}$. Los resultados obtenidos tanto en la caracterización anatómica como físico-mecánica han permitido confirmar la resistencia de las raíces aéreas, refrendando el conocimiento de los pobladores de las comunidades rurales quienes usan las fibras de manera tradicional y ancestral. La calidad de la fibra vegetal de Heteropsis flexuosa presenta posibilidades de competir en el mercado con otras fibras vegetales similares.

PALABRAS CLAVE: Heteropsis flexuosa, anatomía, propiedades fisicomecánicas.

\title{
ANATOMICAL AND PHYSICAL CHARACTERIZATION MECHANICAL OF THE FIBERS OF "TAMSHI" (Heteropsis flexuosa (Kunth) G.S. Bunting) PROVENIENT OF ECHARATE, THE CONVENCION, CUSCO
}

\begin{abstract}
The purpose of this research is to characterize the anatomical structure and physical-mechanical properties of the aerial roots of Heteropsis flexuosa "tamshi wire" (Araceae) exposed to different conditions of humidity and aging treatments. The characteristics of the anatomical elements of the roots confirm the presence of three visibly differentiated zones, observing in the nonperipheral stela a large number of compressed fibers that provide resistance to the roots. Regarding the physical tests, the moisture content of the roots in green condition was $84.9 \%$ and in dry condition to the air it was of $15.9 \%$; the linear density was $65.09 \mathrm{~g} / \mathrm{m}$. The values of the mechanical properties of the roots without aging were lower than those obtained with aging; the elongation was $1.4 \%$ and $0.97 \%$ compared to the initial length, the TEA (tensile energy absorption) was $31138.07 \mathrm{~J} / \mathrm{m} 2$ and $15577.47 \mathrm{~J} / \mathrm{m} 2$, and the torsional strength of $1.065 \mathrm{~kg} / \mathrm{my} 0.945 \mathrm{~kg} / \mathrm{m}$, respectively. Roots with aging showed tensile behavior of $584.78 \mathrm{Kg} / \mathrm{cm} 2$, and without aging of $535.92 \mathrm{Kg} / \mathrm{cm} 2$. The results obtained both in the anatomical and physical-mechanical characterization have allowed to confirm the resistance of the aerial roots, endorsing the knowledge of the inhabitants of the rural communities who use the fibers in a traditional and ancestral way. The quality of the plant fiber of Heteropsis flexuosa presents possibilities to compete in the market with other similar vegetable fibers.

KEYWORDS: Heteropsis flexuosa, anatomy, physicomechanical properties.
\end{abstract}




\section{INTRODUCCIÓN}

Heteropsis es un pequeño género neotropical de la familia Araceae, cuenta entre 12 a 13 especies (Hoffman, 1997). Comprende plantas hemiepífitas lianescentes, con tallos delgados, raíces aéreas largas. Hojas dísticas, coriáceas, enteras, venas primarias numerosas y muy cercanas entre sí, conectadas a través de una vena marginal colectora. La inflorescencia es un espádice erguido, pedunculado (Gentry 1993 y Vásquez 1997). Se distribuye desde la Amazonia brasileña y boliviana hasta el norte de Nicaragua, con centro de diversidad en el sureste de Venezuela y las Guayanas (Hoffman, 1997). La misma fuente indica que las poblaciones de Heteropsis se encuentran en una variedad de tipos de bosques entre 30 a 1000 metros de altitud, incluidos los macizos de las tierras altas y bajas.

Missouri Botanical Garden (2001) precisa que la especie Heteropsis flexuosa cuenta con una amplia distribución en el trópico suramericano, ocurre en Guyana, Guyana Francesa, Suriname, Venezuela, Ecuador, Colombia, Brasil y Perú. En Perú se localiza en la selva amazónica de los departamentos de Amazonas, Loreto, Madre de Dios, Pasco y Puno (Brako \& Zarucchi, 1993). Knab-Vispo et al. (2000) agrega que H. flexuosa no tiene preferencia por algunas especies y tamaño de árboles hospederos, pero si por ciertos tipos de hábitats de bosques no inundados. Sobre el particular, Baluarte y Alván (2001) anotaron cierta preferencia de $H$. flexuosa en árboles hospederos de las familias Chrisobalanaceae y Lecythidaceae en los bosques de terraza alta de Jenaro Herrera.

Según Bunting (1975), Heteropsis flexuosa es una trepadora cuyo tallo es grueso y leñoso, con entre nudos alargados y yemas axilares espiniformes. La hoja tiene un peciolo muy corto y una lámina coriácea angosta-obovada $\mathrm{u}$ oblonga-elíptica con numerosos nervios laterales salientes. La lámina alcanza $27 \mathrm{~cm}$ de largo (incluyendo el largo acumen apical) por $9 \mathrm{~cm}$ de ancho. La inflorescencia mide $6 \mathrm{~cm}$ de largo en antesis, aumentando en maduración hasta $13 \mathrm{~cm}$ de largo. Agrega que las raíces aéreas, partidas en tiras delgadas, se utilizan en lugar de fibras sintéticas y alambre metálico para amarrar. Estas raíces, de $6 \mathrm{~mm}$ o más de diámetro, tienen una superficie castaña y áspera con líneas longitudinales elevadas. Son muy fuertes como indica su nombre vulgar de "alambrito" en Venezuela.

Además de esta denominación, Heteropsis flexuosa recibe diversos nombres vernaculares en los lugares donde crece y es utilizada por los pobladores rurales en la manufactura de artículos utilitarios; no obstante, es sorprendentemente poco estudiada por botánicos y etnobotánicos. Más allá de las descripciones en las obras florísticas, hay ocasionales relatos de especies de Heteropsis en inventarios etnobotánicos, estudios de parcelas etnobotánicas y narrativas de viajes del siglo XIX (Hoffman, 1997).

Los pobladores rurales de la Amazonía peruana utilizan ancestralmente estas raíces aéreas en la construcción de sus viviendas para sujetar las estructuras de madera. También utilizan las raíces de esta especie para manufacturar, cestas, muebles, sombreros y escobas (Baluarte y Del Castillo, 2001).

Las raíces de Heteropsis flexuosa tienen gran demanda en el ámbito rural de la Amazonía peruana. Al respecto, Baluarte y Alván (2002) reportaron el uso de más de 35 mil metros de raíces que ocasionó el descreme de cerca de 50 ha de plantas de Heteropsis flexuosa en los últimos diez años previos al estudio en mención en los bosques aledaños de la localidad de Jenaro Herrera.

La misma fuente agrega que la extracción de las raíces de esta planta hemiepífita se efectúa de manera irracional y consiste en jalar las raíces 
hasta que se rompa del falso tallo, ocasionando muchas veces que esta se desprenda junto con la planta. En el campo el momento oportuno para la cosecha de las raíces se determina por el chasquido característico que emiten las raíces cuando se flexiona con las manos, indicando al poblador local, la madurez de las raíces.

La escasa información sobre las características anatómicas y físico-mecánicas de las raíces aéreas de Heteropsis flexuosa "alambre de tamshi", restringe su uso más amplio y diversificado como materia prima en los mercados nacionales e internacionales. Al respecto, en las dos últimas décadas, se han efectuado algunas investigaciones en Heteropsis flexuosa como los realizados por Hoffman (1997), Saldaña (2004) y Guevara (2011). El primero trató el estudio de las características anatómicas y el segundo además de las características anatómicas, abundó en el estudio de las propiedades físicas de las raíces aéreas; el último de los nombrados efectuó ensayos físicosy mecánicos, pero con metodología diferente a la abordada en el presente estudio.

Sobre el particular, Hoffman (1997) y Saldaña (2004), señalan que la estructura anatómica de la raíz de $H$. flexuosa se caracteriza por presentar cuatro elementos: peridermo, epidermis, corteza y estela; siendo esta última la parte principal de las raíces debido a que cuenta con mayor proporción de fibras muy comprimidas proporcionando mayor resistencia a las raíces. Asimismo, Guevara (2011) indica que $H$. flexuosa presenta contenido de humedad con corteza y sin corteza de $79 \%$ y $129 \%$, respectivamente; agrega que la densidad básica es de $486 \mathrm{~kg} / \mathrm{m} 3$ y $635 \mathrm{~kg} / \mathrm{m} 3$ y la contracción volumétrica de $12,70 \%$ y 5,36\%, con corteza y sin corteza, respectivamente.

En el presente estudio, hemos visto la necesidad de profundizar el estudio de las características anatómicas y físico-mecánicas de las raíces, en particular, simulando las condiciones de envejecimiento en el uso de las raíces para comparar su resistencia natural y proporcionar opciones para la utilización apropiada de las fibras de esta especie. Por lo tanto, el presente estudio contribuye a abordar esta problemática que servirá como fundamento técnico para establecer la confianza en el uso tradicional y más diversificado de las raíces de Heteropsis flexuosa en la Amazonía peruana.

\section{MATERIALES Y METODOS}

\section{LUGAR DE COLECTA DE MUESTRAS DE ALAMBRE TAMSHI}

La colecta de las raíces aéreas de Heteropsis flexuosa "alambre tamshi" se efectuó en la comunidad nativa de Timpia, ubicada en el distrito de Echarate, provincia La Convención, departamento de Cusco; en la parte media del lado oriental de la cordillera del Vilcabamba.

Un total de once plantas que contaban con un mínimo de ocho raíces cada una fueron seleccionadas, de las que se cosecharon 3 raíces para los estudios de laboratorio. Estas plantas estuvieron ubicadas en bosques de transición entre los bosques montanos y los bosques de la parte baja de la Amazonía peruana, entre los 450 a $3450 \mathrm{msnm}$, a $241,5 \mathrm{~km}$ del centro poblado principal cuyas coordenadas UTM 3737146,100 E, 8663467,000 N.

\section{NORMAS TÉCNICAS PARA LA EJECUCIÓN DE LOS ENSAYOS}

La caracterización anatómica, física y mecánica se realizó usando las siguientes normas técnicas:

- Norma de Procedimientos em Estudos de Anatomia de Madeira: I, Angiospermae del IBAMA (1991) y la List of Microscopic Features for Hardwood Identification del IAWA (1989). Estas normas establecen los procedimientos para el estudio y la descripción de la estructura anatómica y la identificación de la madera basados en este aspecto. 
- Densidad lineal (Denier), se realizó de acuerdo con la norma TAPPI T411 (1997a). Una unidad de denier corresponde a la masa (g) de $9000 \mathrm{~m}$ lineales de la fibra, cuanto más alto es el Denier, más gruesa es la sección de la fibra, del mismo modo, la densidad lineal está relacionada con el peso específico de los componentes de la fibra.

- Contenido de humedad, se hizo uso de la norma TAPPI T412 om-94 (1997b). El contenido de humedad se refiere a la propiedad de un material para acumular agua en sus elementos vasculares. Esta propiedad influye mucho en su peso a la vez que afecta otras propiedades físicas (peso específico, contracción o hinchamiento de sus dimensiones), las propiedades de resistencia mecánica y de resistencia al ataque de hongos e insectos xilófagos.

- Coloración, se hizo uso de la Tabla Munsell. El color es una percepción humana de la luz reflejada por un objeto; es un atributo de apariencia de los productos y su observación permite detectar ciertas anomalías y defectos.

- Tensión, se usó la norma TAPPI 494 om-96 (1996a). Mide la capacidad de una muestra para resistir fuerzas que tratan de estirar la fibra, por lo general en dirección longitudinal. La resistencia a la tensión puede estar afectada por el ancho del lumen y el ancho de la fibra, medidas a través del coeficiente de flexibilidad, y también existe una influencia negativa al aumentar el espesor de pared, no habiendo influencia significativa de la longitud de la fibra.

- Tensile energy absorpyion o absorción de energía de tracción (TEA), se usó la norma TAPPI T484 om-96 (1996b). Es el trabajo realizado cuando una muestra es sometida a la ruptura en tracción; mide la capacidad para absorber energía e indica la durabilidad del material cuando se le somete a esfuerzos respectivos, la TEA se expresa como energía por unidad de área del espécimen de prueba, además lo define como el área bajo la curva de carga-elongación, en relación con la superficie de papel entre mordazas; este resultado se utiliza normalmente para caracterizar la capacidad de absorción de energía de una muestra, en este caso de la fibra .

- Torsión, se usó de la norma NTE INEN 0140. Los ensayos de torsión se realizan para determinar las propiedades mecánicas de un objeto o una muestra cuando se le aplican fuerzas de corte. Las muestras para ensayos de torsión (normalmente con sección transversal circular) se enganchan por los extremos a los soportes de la máquina de ensayos y se giran hacia lados contrarios desde sus extremos, produciéndose fuerzas de corte hasta la rotura de la muestra, siendo la resultante de fuerzas paralelas de igual magnitud, pero en sentido opuesto en el mismo sólido, se caracteriza geométricamente porque cualquier curva paralela al eje de la pieza deja de estar contenida en el plano formado inicialmente por las dos curvas, en lugar de eso una curva paralela al eje se retuerce alrededor de sí mismo.

- Análisis del envejecimiento, se usó la norma TAPPI- T544 sp-97 (1997c). Consiste en evaluar la resistencia de un material puesto en uso, en este caso simulando condiciones de envejecimiento para probar como influye estas características en la resistencia del material cuando es sometido a una fuerza o carga que tiende a alterar su consistencia.

Estas características anatómicas y los ensayos para la determinación de las propiedades físicomecánicas de las raíces de Heteropsis flexuosa se realizaron en los Laboratorios de Anatomía de la Madera y Pulpa y Papel de la facultad de Ciencias Forestales de la Universidad Nacional Agraria La Molina (UNALM). 


\section{DISEÑO EXPERIMENTAL}

Las raíces aéreas fueron cortadas en segmentos de diferentes longitudes, tomando como referencia las raíces cosechadas cerca de la planta, cerca al punto de inserción de la raíz en el suelo y en una posición media. Se utilizó un diseño completamente al azar (DCA) con arreglo factorial de $2 \times 2$, con 10 repeticiones para los ensayos de tensión, alargamiento y TEA.

Para los ensayos de torsión, utilizamos tres repeticiones, cuyas variables independientes fueron dos niveles de contenido de humedad (raíces húmedas y raíces secas al aire por 48 horas hasta conseguir peso constante) y dos niveles de envejecimiento (con envejecimiento y sin envejecimiento), y las variables dependientes fueron el módulo de ruptura de las fibras (MOR), el módulo de elasticidad de las fibras (MOE), torsión de las fibras, y la Absorción de energía de tracción (TEA).

\section{RESULTADOS}

\section{CARACTERIZACIÓN ANATÓMICA}

En la figura 1 se observa el corte transversal de la raíz aérea seca de Heteropsis flexuosa, donde se nota tres zonas claramente diferenciadas que se describen a continuación.

\section{ZONA A : ANILLO ESCLERENQUIMAL CIRCUNESTELAR}

En esta zona se observa que los extremos de las celdas de las fibras van disminuyendo de diámetro, generalmente tienen forma curva. En la sección transversal, las fibras se encuentran estrechamente agrupadas a manera de paquetes con poco espacio intracelular visible, cuya dimensión es de 190 a $870 \mu$ m de longitud total y ancho de 9 a $36 \mu \mathrm{m}$, las paredes celulares son engrosadas uniformemente con capas laminadas claramente visibles (Figura 1).

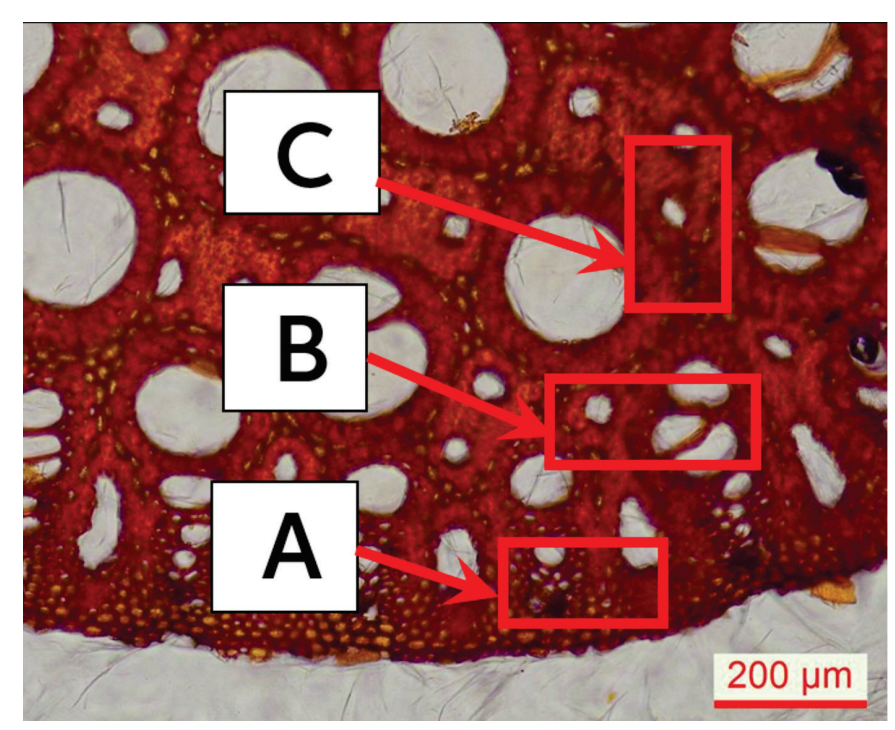

Figura 1. Diferenciación de 3 regiones en corte transversal de las raíces aéreas de Heteropsis flexuosa cosechadas en Echarate, Cusco. A: Anillo esclerenquimal circunscrito, B: Estela periférica, C: Estela no periférica.

\section{ZONA B: ESTELA PERIFÉRICA}

Las fibras en la estela periférica tienen una longitud media de $3240 \mu \mathrm{m}$ y ancho promedio de $22 \mu \mathrm{m}$ (Figura 2).

En la sección transversal las fibras son cuadrangulares a hexagonales y ligeramente redondeadas en su contorno. En la célula las paredes son de espesor uniforme y se aprecian visualmente en las láminas, la mayoría de las fibras maceradas se mostraron erguidas y rígidas, siendo algunas flexibles y curvas bajo el microscopio. Los extremos de la célula de las fibras se van estrechando de manera acicular, ocasionalmente doblados, agudamente puntiagudos en un extremo y ligeramente redondeados en el extremo opuesto.

\section{ZONA C: ESTELA NO PERIFÉRICA}

Las fibras en la estela no periférica son idénticas a las de la estela periférica, con una ligera diferencia en tamaño, cuenta con una longitud de $1500 \mu \mathrm{m}$ y ancho medio de $21 \mu \mathrm{m}$. 

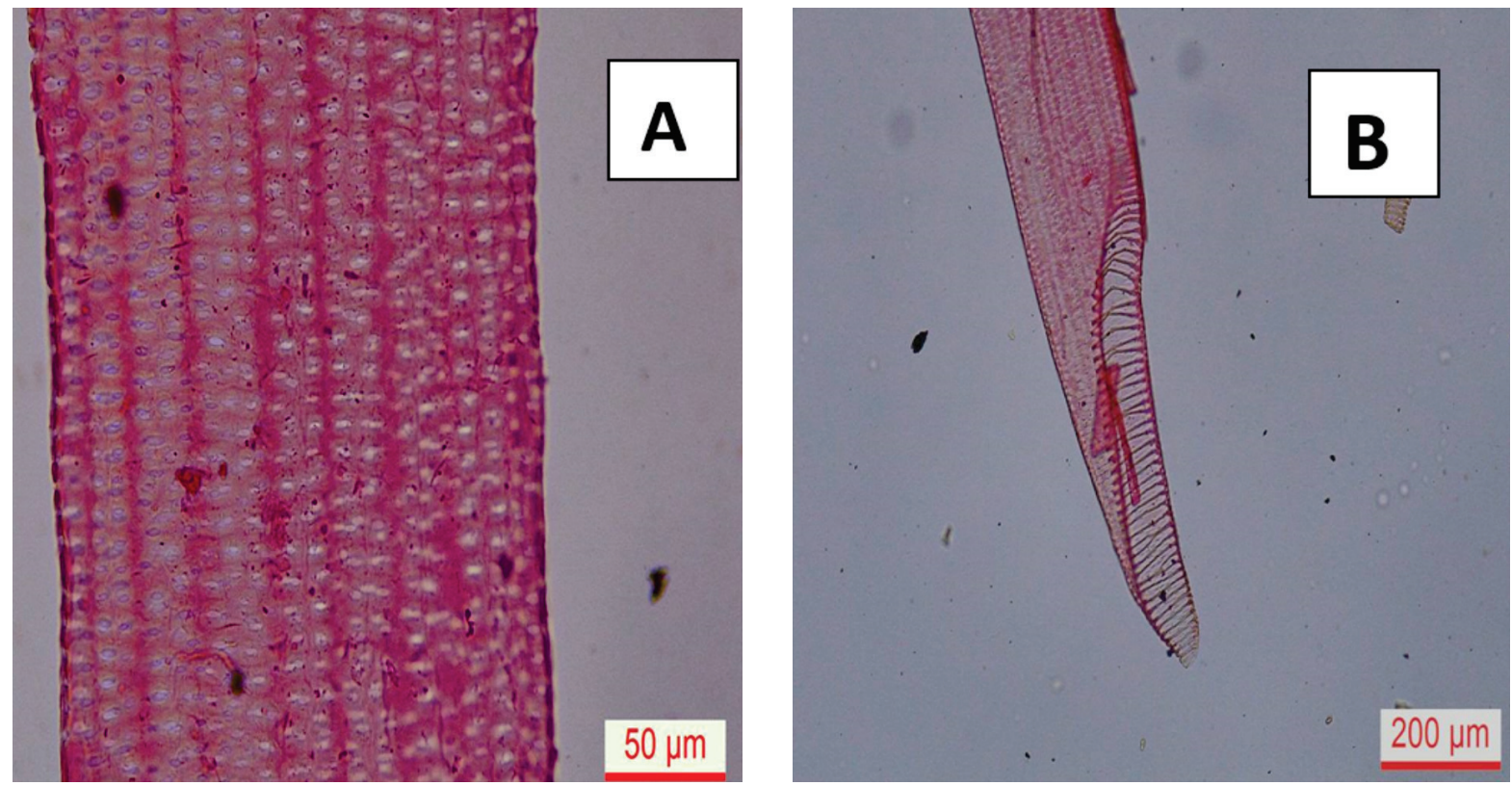

Figura 2. Elementos anatómicos de los vasos de las raíces aéreas de Heteropsis flexuosa: A: punteaduras simples, B: placas de perforación escaleriformes.

En cuanto ala longitud de los vasos del metaxilema como del protoxilema en promedio es de $1193 \mu \mathrm{m}$ presentando punteaduras simples con disposición opuesta, distribución escaleriforme y placas de perforación escaleriformes con 33-49 barras de engrosamiento con orientación oblicua (Figura 2).

En el corte transversal de la raíz, se muestra la dispersión de los vasos resaltando dos zonas que cambian gradualmente en densidad de vasos. Obtuvimos una densidad de 9 vasos $/ \mathrm{mm}^{2}$ en la zona A hasta 16 vasos $/ \mathrm{mm}^{2}$ en la zona B conforme va de la zona periférica al centro.

Las fibras mostraron un diámetro promedio de 22,366 $\mu \mathrm{m}$ y la longitud promedio de las fibras fue de $3243,92 \mu \mathrm{m}$; mientras que la longitud de los vasos fue de 1193,17 $\mu \mathrm{m}$ (Tabla 1).

El diámetro promedio de los poros grandes fue de 151,96 $\mu \mathrm{m}$, mientras que el diámetro promedio de los poros pequeños fue de $37,81 \mu \mathrm{m}$ (Tabla 2). 
Tabla 1. Valores promedios de los elementos xilemáticos de las raíces aéreas de Heteropsis flexuosa

\begin{tabular}{|c|c|c|c|c|c|c|}
\hline \multirow[b]{2}{*}{ Individuo } & \multirow[b]{2}{*}{ Sección } & \multicolumn{4}{|c|}{ Fibras } & \multirow[b]{2}{*}{ Vasos } \\
\hline & & $\begin{array}{l}\text { Diámetro } \\
\text { (micras) }\end{array}$ & $\begin{array}{c}\text { Espesor de } \\
\text { pared (micras) }\end{array}$ & $\begin{array}{l}\text { Lumen } \\
\text { (D-2E) }\end{array}$ & $\begin{array}{l}\text { Longitud } \\
\text { (micras) }\end{array}$ & \\
\hline \multirow{3}{*}{1} & Apical & 21,32 & 6,94 & 7,44 & 3191 & 1183 \\
\hline & Media & 21,69 & 6,66 & 8,37 & 3123 & 1181 \\
\hline & Baja & 24,00 & 8,39 & 7,21 & 3642 & 978 \\
\hline \multirow{3}{*}{2} & Apical & 22,93 & 7,14 & 8,65 & 3372 & 1148 \\
\hline & Media & 22,68 & 5,87 & 10,95 & 2959 & 1093 \\
\hline & Baja & 21,98 & 6,84 & 8,30 & 2904 & 1075 \\
\hline \multirow{3}{*}{3} & Apical & 21,04 & 7,28 & 6,47 & 3015 & 1297 \\
\hline & Media & 21,87 & 7,93 & 6,01 & 3305 & 1489 \\
\hline & Baja & 24,09 & 9,47 & 5,15 & 3091 & 1136 \\
\hline \multirow{3}{*}{4} & Apical & 24,12 & 9,97 & 4,19 & 3120 & 1153 \\
\hline & Media & 16,13 & 7,58 & 0,98 & 3320 & 1122 \\
\hline & Baja & 24,55 & 8,06 & 8,42 & 3245 & 1118 \\
\hline \multirow{3}{*}{5} & Apical & 24,12 & 9,97 & 4,19 & 3120 & 1153 \\
\hline & Media & 20,84 & 7,51 & 5,82 & 4188 & 1619 \\
\hline & Baja & 24,14 & 9,97 & 4,20 & 3067 & 1153 \\
\hline \multicolumn{2}{|c|}{ X Total } & 22,366 & 7,972 & 6,423 & 3243,92 & 1193,17 \\
\hline \multicolumn{2}{|c|}{ X Apical } & 22,706 & 8,260 & 6,188 & 3163,00 & 1186,00 \\
\hline \multicolumn{2}{|c|}{ X Media } & 20,632 & 7,110 & 6,426 & 3379,00 & 1300,00 \\
\hline \multicolumn{2}{|c|}{ X Basal } & 23,752 & 8,546 & 6,656 & 3189,00 & 1092,00 \\
\hline
\end{tabular}


Tabla 2. Valores promedio de los poros de Heteropsis flexuosa

\begin{tabular}{|c|c|c|c|c|c|}
\hline \multirow[b]{3}{*}{ Individuo } & \multirow[b]{3}{*}{ Sección } & \multicolumn{4}{|c|}{ Poros } \\
\hline & & \multicolumn{2}{|c|}{ Grandes } & \multicolumn{2}{|c|}{ Pequeños } \\
\hline & & $\begin{array}{l}\text { Diámetro } \\
\text { (micras) }\end{array}$ & Por $\mathrm{mm}^{2}$ & $\begin{array}{l}\text { Diámetro } \\
\text { (micras) }\end{array}$ & Por $\mathrm{mm}^{2}$ \\
\hline \multirow{3}{*}{1} & Apical & 158 & 13 & 35 & 17 \\
\hline & Media & 165 & 13 & 37 & 17 \\
\hline & Baja & 150 & 15 & 34 & 16 \\
\hline \multirow{3}{*}{2} & Apical & 146 & 11 & 34 & 16 \\
\hline & Media & 146 & 10 & 34 & 15 \\
\hline & Baja & 142 & 12 & 37 & 14 \\
\hline \multirow{3}{*}{3} & Apical & 139 & 10 & 42 & 14 \\
\hline & Media & 147 & 14 & 41 & 17 \\
\hline & Baja & 143 & 16 & 49 & 17 \\
\hline \multirow{3}{*}{4} & Apical & 137 & 9 & 37 & 17 \\
\hline & Media & 151 & 10 & 33 & 13 \\
\hline & Baja & 186 & 11 & 43 & 15 \\
\hline \multirow{3}{*}{5} & Apical & 163 & 9 & 37 & 14 \\
\hline & Media & 155 & 10 & 37 & 17 \\
\hline & Baja & 150 & 9 & 36 & 13 \\
\hline \multicolumn{2}{|c|}{ X Total } & 151,96 & 11 & 37,81 & 15 \\
\hline \multicolumn{2}{|c|}{ DS } & 12,50 & 2,26 & 4,34 & 1,55 \\
\hline \multicolumn{2}{|c|}{$C V$} & 8,23 & 0,2 & 0,11 & 0,1 \\
\hline \multicolumn{2}{|c|}{ X Apical } & 148,6 & - & 37 & - \\
\hline \multicolumn{2}{|c|}{ X Media } & 152,8 & - & 36 & - \\
\hline \multicolumn{2}{|c|}{ X Basal } & 154,2 & - & 40 & - \\
\hline
\end{tabular}




\section{CARACTERIZACIÓN DE LAS PROPIEDADES FÍSICAS}

\section{Contenido de humedad y coloración}

Las raíces colectadas en condición verde presentaron en promedio contenido de humedad de $84,88 \%$, mientras que cuando fueron secadas en condiciones de ambiente fue de $15,9 \%$.

La fibra de $H$. flexuosa sin corteza presenta una coloración que obedece al código 5 Y 8/6 correspondiendo a color claro medio amarillo (figura 3).

\section{Densidad lineal (denier)}

El menor valor para el denier es de $560800 \mathrm{~g} / 9000 \mathrm{~m}$ y el mayor valor es de $610700 \mathrm{~g} / 9000 \mathrm{~m}$.

\section{Caracterización de las propiedades mecánicas}

La resistencia máxima a la tracción para los haces fibrosos de la fibra de $H$. flexuosa en estado seco al aire sin ser sometido al envejecimiento acelerado, es en promedio $616,9 \mathrm{~kg} / \mathrm{cm}^{2}$ $(7,45 \mathrm{kp})$, mientras que en estado saturado (65\% de humedad) disminuye su resistencia a 535,92 $\mathrm{kg} / \mathrm{cm}^{2}$ (6,4 kp), las muestras ensayadas luego de un proceso de envejecimiento presentan

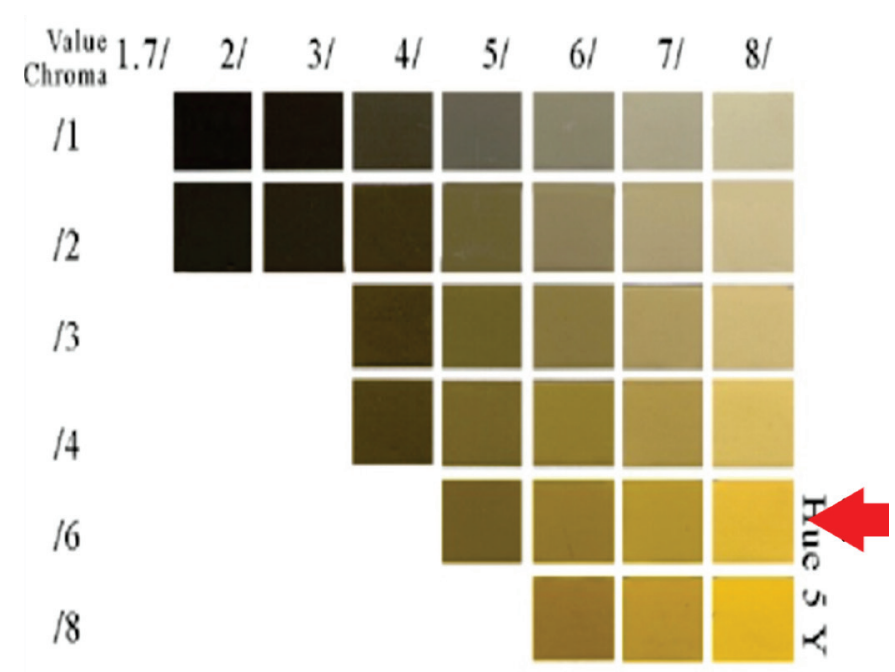

Figura 3. Tabla Munsell. Obsérvese que la flecha indica el color de la fibra de $H$. flexuosa.

menor resistencia alcanzando $584,78 \mathrm{~kg} / \mathrm{cm}^{2}$ en condiciones de saturación de humedad (80\%).

Las muestras secas al aire presentan el menor grado de elongación correspondiéndole $0,74 \%$ a $15 \%$ de humedad, seguido de la muestra sometida a envejecimiento con $0,97 \%$ a $80 \%$ de humedad y finalmente la muestra en condición verde con $1,4 \%$ a $65 \%$ de humedad (Tabla 3 ).

La absorción de energía de tracción de material en láminas de $H$. flexuosa en estado seco (15\%) sin ser sometidas a envejecimiento resultó en promedio de $31138 \mathrm{~J} / \mathrm{m}^{2}$ (Tabla 3)

Tabla 3. Valores promedios de propiedades mecánicas de tracción, alargamiento TEA y torsión bajo dos condiciones de humedad y envejecimiento

\begin{tabular}{|c|c|c|c|c|c|}
\hline \multirow{3}{*}{$\begin{array}{c}\text { Propiedades } \\
\text { Mecánicas }\end{array}$} & \multirow{3}{*}{ Unidad } & \multicolumn{3}{|c|}{ Promedio } & \multirow{3}{*}{$\begin{array}{l}\text { Coeficiente de } \\
\text { Variabilidad (\%) }\end{array}$} \\
\hline & & \multicolumn{2}{|c|}{ SE } & \multirow{2}{*}{$\frac{\mathrm{CE}}{\mathrm{CH} 80 \%}$} & \\
\hline & & $\mathrm{CH} 15 \%$ & $\mathrm{CH} 60 \%$ & & \\
\hline Tracción & $\mathrm{Kg} / \mathrm{cm}^{2}$ & 616,9 & 535,92 & 584,78 & 9,75 \\
\hline Alargamiento & $\%$ & 0,74 & 1,4 & 0,97 & 22,54 \\
\hline TEA & $\mathrm{J} / \mathrm{m}^{2}$ & 31138 & 200978 & 15577 & 31,083 \\
\hline Torsión & $\mathrm{Kg} / \mathrm{m}$ & 1,29 & 1,065 & 0,945 & 18,745 \\
\hline
\end{tabular}

SE: Muestras sin efecto de envejecimiento

CE: Muestras con efecto de envejecimiento

$\mathrm{CH}$ : Contenido de humedad 
La torsión de material en láminas de fibras de H. flexuosa sin efecto de envejecimiento $(15 \%$ de contenido de humedad) es en promedio de $1,29 \mathrm{~kg} / \mathrm{m}$, seguido de la muestra que fue sometida a envejecimiento $(65 \%$ de contenido de humedad) con promedio de $1,065 \mathrm{~kg} / \mathrm{m}$ y en estado verde ( $80 \%$ de contenido de humedad) presenta promedio de $0,945 \mathrm{~kg} / \mathrm{m}$.

\section{Influencia del envejecimiento en las propiedades mecánicas}

En la tabla 4, se muestra que las propiedades En la tabla 4, se muestra que las propiedades mecánicas de las fibras de $H$. flexuosa es significativo para los ensayos de tracción, alargamiento, TEA y torsión $(<0,0001)$.

En la Tabla 5 se muestra la significancia estadística de las medias de las propiedades de tracción, alargamiento, TEA y torsión de las fibras de $H$. flexuosa con respecto al efecto del contenido de humedad y envejecimiento.

En la Figura 4, se observa los valores medios y la variabilidad del error muestral de las propiedades físico mecánicas como Torsión, TEA, Alargamiento y Resistencia a la tracción simulando condiciones de envejecimiento y sin envejecimiento con diferentes contenidos de humedad de las fibras. Al respecto, los valores de torsión sin envejecimiento en condición secaalaire son mayores que las medias con envejecimiento y sin envejecimiento con fibras saturadas de humedad. La media de TEA sin envejecimiento en condición de saturación de la fibra es mayor que las medias con y sin envejecimiento tanto en condición anhidra como saturada de humedad, inclusive es superior a la media de la TEA sin envejecimiento; sin embargo, la variabilidad del error muestral es bajo en todos los casos ensayados. En relación al alargamiento de la fibra el comportamiento es diferente, en el cual se observa que la media de los valores con y sin envejecimiento en condiciones de saturación de humedad son muy semejantes; situación similar se observa en las muestras con envejecimiento y sin envejecimiento en condiciones anhidras. La resistencia a la tracción con y sin envejecimiento muestra medias muy similares en condiciones de saturación de humedad, a diferencia de este la media de la resistencia a la tracción con y sin envejecimiento en condición de humedad seca al aire presenta resultados significativos en la prueba de Tukey.

\section{DISCUSIÓN}

\section{CARACTERIZACIÓN ANATÓMICA}

La estructura anatómica de la raíz aérea de $H$. flexuosa está conformada por tres zonas claramente diferenciadas, este hallazgo es consistente con otros estudios realizados anteriormente como por ejemplo Hoffman (1997) y Saldaña (2004). El tamaño de los elementos vasculares observados en este estudio guarda mucha relación con lo observado en los estudios antes mencionados. Asimismo, la presencia de un mayor número de fibras comprimidas en la zona "C" donde está ubicada la estela no periférica confiere mayor resistencia a las raíces, tal como fue observado por Hoffman (1997). Los elevados valores anotados en tracción y torsión de las fibras de $H$. flexuosa están relacionadas con esta característica.

La cantidad de poros grandes y pequeños aumenta en número en la parte central y con ello aumenta la cantidad de espacios que serán ocupados por fluidos, encontrándose a su vez menor cantidad de fibras compactas, en consecuencia, las propiedades mecánicas se ven disminuidas, debido a que las fibras poseen componentes que le proporcionan mayor resistencia; por otro lado, al existir espacios vacíos, esto le otorga al material mayor módulo de elasticidad, la gran capacidad para retener agua, explicaría por qué 
Tabla 4. Valores de Análisis de Varianza para las propiedades mecánicas de tracción, Alargamiento, TEA y torsión

\begin{tabular}{|c|c|c|c|c|c|c|c|c|c|c|c|c|c|}
\hline \multirow{2}{*}{$\begin{array}{l}\text { Fuente de } \\
\text { Variabilidad }\end{array}$} & \multirow[b]{2}{*}{ G,L, } & \multicolumn{3}{|c|}{ Tracción } & \multicolumn{3}{|c|}{ Alargamiento } & \multicolumn{3}{|c|}{ TEA } & \multicolumn{3}{|c|}{ Torsión } \\
\hline & & $\begin{array}{l}\text { Valor } \\
\text { de F }\end{array}$ & $\operatorname{Pr}>\mathrm{F}$ & Sig & $\begin{array}{l}\text { Valor } \\
\text { de F }\end{array}$ & $\operatorname{Pr}>\mathrm{F}$ & Sig & $\begin{array}{l}\text { Valor } \\
\text { de F }\end{array}$ & $\operatorname{Pr}>\mathrm{F}$ & Sig & $\begin{array}{l}\text { Valor } \\
\text { de F }\end{array}$ & $\operatorname{Pr}>\mathrm{F}$ & Sig \\
\hline $\mathrm{H}$ & 1 & 22,43 & $<0,0001$ & * & 29,37 & $<0,0001$ & * & 11,39 & 0,0022 & $* *$ & 58,46 & $<0,0001$ & ** \\
\hline E & 1 & 364,8 & $<0,0001$ & $* *$ & 182,0 & $<0,0001$ & $* *$ & 16,23 & $<0,0001$ & $* *$ & 251,2 & $<0,0001$ & $* *$ \\
\hline$H^{*} E$ & 1 & 9,47 & 0,0046 & * & 0,47 & 0,0204 & NS & 1,08 & 0,2247 & NS & 1,24 & 0,2099 & NS \\
\hline Error & 40 & - & - & - & - & - & - & - & - & - & - & - & - \\
\hline Total & 43 & - & - & - & - & - & - & - & - & - & - & - & - \\
\hline$C V$ & - & & 17,43 & & & 27,94 & & & 34,84 & & & 31,93 & \\
\hline
\end{tabular}

$\mathrm{H}$ : Efecto del factor contenido de humedad

E: Efecto del factor envejecimiento

$H^{*} E$ : Efecto entre la interacción del contenido de humedad y el envejecimiento

CV: Coeficiente de variabilidad; NS: no significativo; $\left(^{*}\right)$ significativo; $\left({ }^{*}\right)$ altamente significativo

Sig: Significancia

Tabla 5. Valores de la prueba de Tukey para evaluación las propiedades mecánicas de tracción, alargamiento, torsión y TEA, para humedad y envejecimiento

\begin{tabular}{lcccccc}
\hline \multirow{2}{*}{ Propiedades Mecánicas } & \multicolumn{3}{c}{ Humedad } & & \multicolumn{3}{c}{ Envejecimiento } \\
\cline { 2 - 7 } & Nivel & Media & Tukey & Nivel & Media & Tukey \\
\hline \multirow{2}{*}{ Tracción $\left(\mathrm{kg} / \mathrm{cm}^{2}\right)$} & $18 \%$ & 243,01 & B & SE & 512,66 & A \\
& $80 \%$ & 356,97 & A & CE & 87,32 & B \\
Alargamiento (\%) & $18 \%$ & 1,27 & B & SE & 3,211 & A \\
TEA $\left(\mathrm{J} / \mathrm{m}^{2}\right)$ & $80 \%$ & 2,46 & A & CE & 0,52 & B \\
& $18 \%$ & 445,72 & B & SE & 1046,82 & A \\
Torsión $(\mathrm{kg} / \mathrm{m})$ & $80 \%$ & 666,48 & A & CE & 65,39 & B \\
& $18 \%$ & 0,48 & B & SE & 1,13 & A \\
\hline
\end{tabular}

SE: Muestras si efecto de envejecimiento

CE: Muestras con efecto de envejecimiento 
a) Torsión

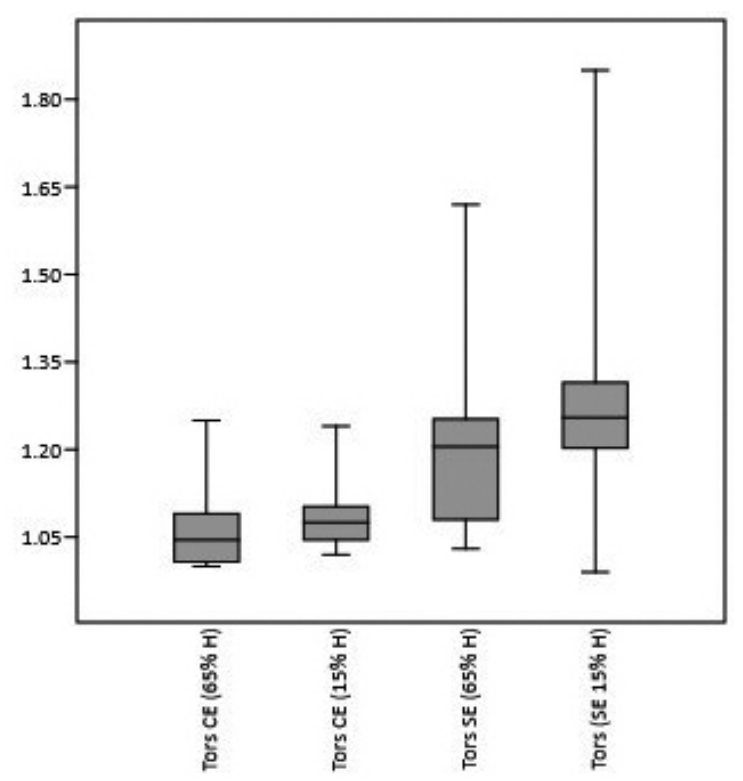

c) Alargamiento

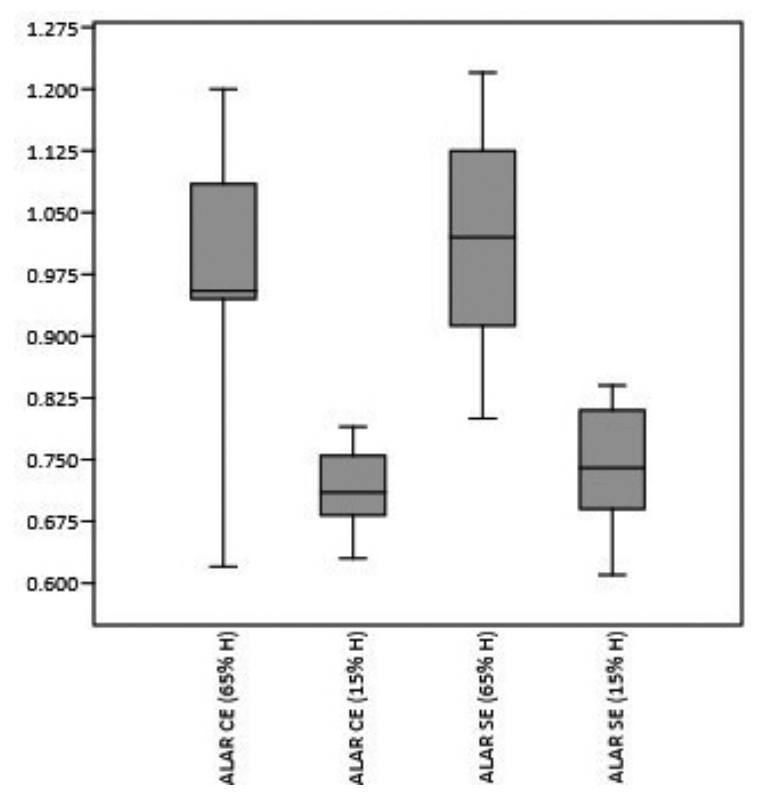

b) TEA

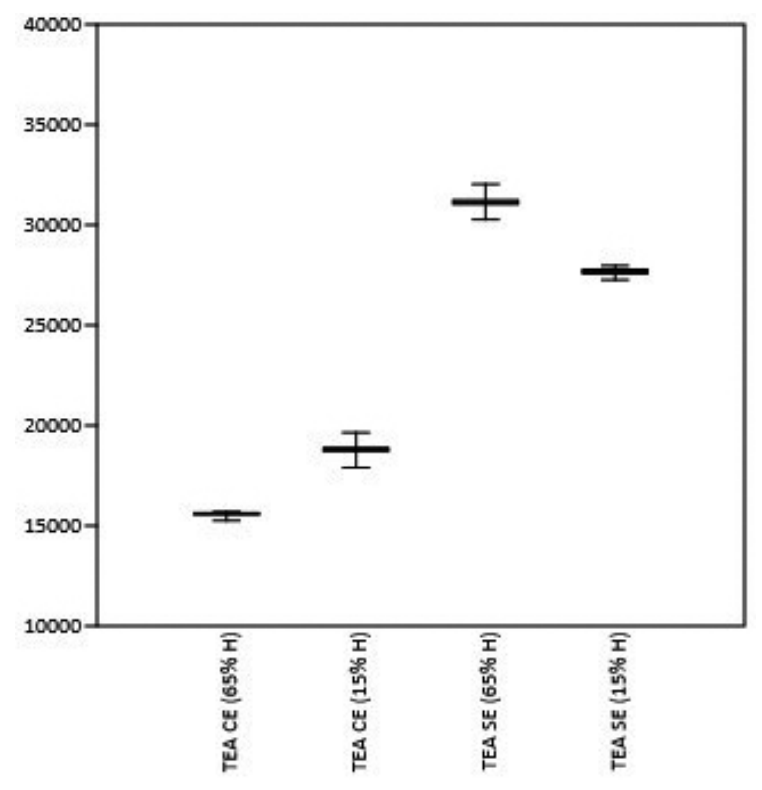

d) Resistencia a la Tracción

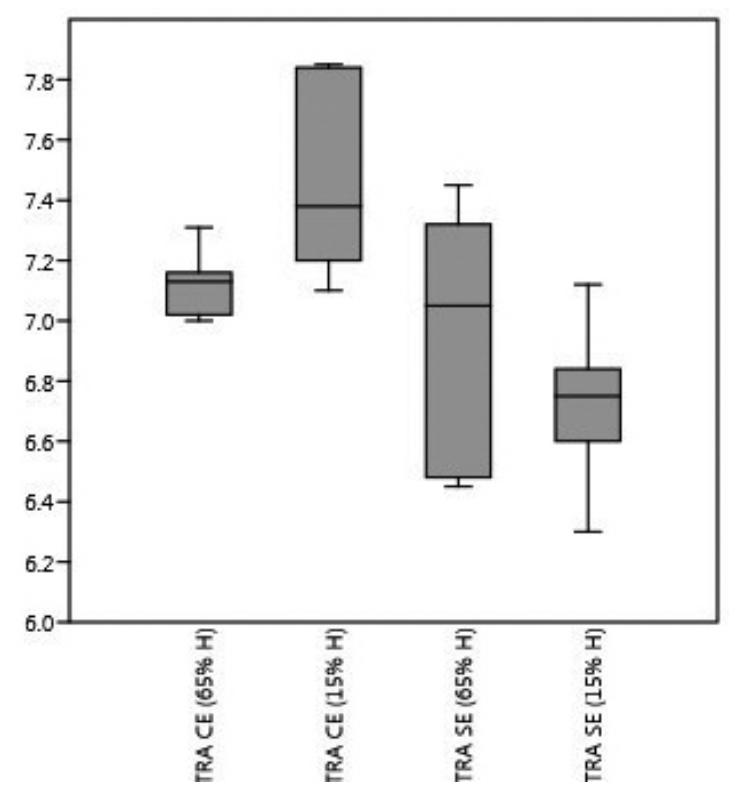

Figura 4: Valores medios y error estándar en: a) Torsión, b) TEA, c) Alargamiento y d) Resistencia a la tracción con muestras sometidas a tratamiento de envejecimiento (CE) y sin envejecimiento (SE) con diferentes contenidos de humedad (15 y 65\%). 
presenta mayor módulo de elasticidad; ello es el resultado de la existencia de paredes delgadas y cavidades celulares relativamente grandes esto significa que en su estructura anatómica concentra gran cantidad de agua, tal como fue anotado por Guevara (2011).

Por otro lado, las variaciones observadas en la densidad Denier dependen de la ubicación del material vegetal respecto a la longitud de la raíz, las muestras extraídas de la parte inferior de la raíz presentan menor densidad debido que el material vegetal está poco constituido, ello quiere decir que la densidad lineal de los materiales lignocelulósicos está condicionada con la madurez del tejido.

\section{PROPIEDADES FÍSICAS}

Las muestras húmedas de raíces de $H$. flexuosa arrojaron $84.88 \%$ de humedad, aproximándose mucho al valor de $80 \%$ reportado por Guevara (2011). Asimismo, se observaron pequeñas diferencias porcentuales entre individuos de la misma especie, esto se debe a que el contenido de humedad tiende a mostrar mayor variación entre región de procedencia o época del año, tal como afirma Diaz et al, (2011) para el caso de muestras de madera.

El contenido de humedad obtenido con $H$. flexuosa en este estudio contrasta con otras especies, por ejemplo Guevara (2011) menciona que la especie Desmoncus polyacanthos, presenta contenido humedad de 191\%, lo cual se explica por abundante parénquima en su estructura anatómica dando la apariencia a un material corchoso en el cual se concentraría gran cantidad de agua; asimismo Thoracocarpus bissectus "cesto tamshi" muestra contenido de humedad superior a $200 \%(\mathrm{CH}=244 \%)$. Estos porcentajes elevados de contenido de humedad hacen referencia a una madera de baja densidad, cuya estructura anatómica está constituida en mayor proporción por células de paredes delgadas con cavidades celulares grandes, confiriéndole la propiedad de retener más de su propio peso en agua según JUNAC (1989).

\section{PROPIEDADES MECÁNICAS}

Con respecto al comportamiento de las fibras de $H$. flexuosa a la tracción sin envejecimiento, nuestro resultado es superior al encontrado por Guevara (2011) quien reportó valor equivalente a 257,6 $\mathrm{kg} / \mathrm{cm}^{2}$ para la misma especie, sobre el particular, la misma fuente sostiene que se producen cambios en las propiedades mecánicas de las especies con la edad, teniendo la capacidad de convertirse en partes vegetales más resistentes cuando cuentan con mayores longitudes, produciéndose aumento de lignificación de las células; sin embargo, en este caso, se debe tener en consideración que se desconoce la edad de los individuos.

La tasa de absorción de energía (TEA) es una propiedad que mide la capacidad para absorber energía que tiene el material e indica el tiempo de vida útil o la durabilidad del material cuando se somete a fuerzas consecutivas. Valores altos en el TEA, significaría que se requiere una mayor cantidad de energía para que las fibras se rompan, por tal motivo, se debe contemplar los valores de TEA para analizar el uso del material. Por su parte la torsión es importante porque es determinante en la capacidad de trenzado y/o fibrilado, para la producción de cestería, mueblería, amarres con fines estructurales, etc. En nuestro caso, las muestras de TEA sin envejecimiento presentan mayores valores comparado con las muestras sometidas a pruebas de envejecimiento, independientemente del contenido de humedad; alternativamente, las muestras que fueron sometidas a envejecimiento muestran mayor TEA cuando no están húmedas.

Al realizar las comparaciones de las medias del efecto del envejecimiento sobre las propiedades mecánicas mediante la prueba estadística de Tukey se comprueba que las muestras en estado 
saturado presentan menores valores comparado con las muestras que estuvieron secas al aire.

Dentro de las propiedades mecánicas bajo efecto del envejecimiento acelerado o intemperismo provocó que la resistencia disminuya de $616,9 \mathrm{~kg} / \mathrm{cm} 2$ a $584,74 \mathrm{~kg} / \mathrm{cm}^{2}$ en cuanto al porcentaje de alargamiento o elongación incrementó de 0,74 a $0,97 \%$, por otro lado, la energía absorbida generada por la tensión (TEA) disminuyó de 31138 a 15577 J/cm², por último, para el ensayo de torsión el valor se vio reducido de 1,29 a $1,06 \mathrm{~kg}$

El rol que cumple el contenido de humedad en las muestras es importante, las variaciones afectaran directamente las propiedades mecánicas como se puede observar en las diferentes pruebas que se sometieron; al respecto, Arroyo (1983) menciona que la variación en el contenido de humedad, causada por la dispersión de la sustancia sólida en la pared celular, produce cambios en las propiedades de resistencia del material.

En la especie $H$. flexuosa el incremento de humedad produjo un decremento en las propiedades mecánicas específicamente la resistencia a la tracción, sin embargo, en alargamiento el porcentaje aumentó.

Según Arroyo (1983) los cambios a causa del envejecimiento (efectos de la temperatura y humedad) se deben principalmente a la hidrólisis de la celulosa, es decir, una disminución en la proporción de celulosa por acción del agua, añade también que los que mayor efecto tendrán en el "envejecimiento acelerado" serán los que poseen altos niveles de carbohidratos como el parénquima, debido a que los azucares sometidos a las condiciones de altas temperaturas y alto porcentaje de humedad, como consecuencia se provoca la reacción de hidrolisis, generando un medio alcalino.

\section{CONCLUSIONES}

- Existe una relación directa entre la resistencia mecánica y la presencia de numerosas fibras comprimidas en la estela no periférica observada en la sección transversal de las raíces de $H$. flexuosa.

- Las propiedades mecánicas de las fibras de H. flexuosa confirman su alta resistencia, resultando superior a otras fibras similares, lo que ratifica su preferencia por los pobladores rurales para sujetar las estructuras de madera de viviendas rurales.

- Las propiedades físico-mecánicas de las fibras sometidas a pruebas de envejecimiento resultaron inferiores al material sin envejecimiento, lo que revela que las fibras reducen su resistencia con el paso del tiempo.

\section{BIBLIOGRAFÍA CITADA}

Arroyo, J. 1983. Propiedades físico-mecánicas de la madera. Universidad de los Andes, Facultad de Ciencias Forestales, Departamento de Publicaciones. Mérida, Venezuela. 26-43 p.

Baluarte, J. y Alván, G. 2002. Ecología, uso e impacto de la extracción de "alambre tamshi" Heteropsis flexuosa en Jenaro Herrera. En resúmenes IX Congreso Nacional de Botánica. Iquitos, Perú.

Baluarte, J. y Del Castillo, D. 2001. Tamshi: otro producto no maderable de los bosques amazónicos con importancia económica. Folia Amazónica V 12(1-2):155-160.

Brako, L, \& Zarucchi, J.L. 1993. Catalogue of the flowering plants and gymnosperms of Peru. Missouri Botanical Garden, Missouri. Pp. 1286 Bunting, G. 1975. Sinopsis de las Araceae de Venezuela. Instituto de Botánica Agrícola, Facultad de Agronomía, Universidad Central 
de Venezuela. Maracaibo, Venezuela. En: Agronomía Tropical 10(1-4):139-290.

Diaz, M.; Pecho, R.; Guarda, D. 2011. Variación de las propiedades físicas de Calycophyllum spruceanum (Benthan) Hooker F. ex Chuman (Capirona) de una plantación de ocho años en la zona de Tingo María, Perú. Universidad Nacional Agraria de la Selva. 18 p. (http://www,cnf,org,pe/secretaria conflat/memorias/DOCUMENTO\%20MESAS/ MESA\%203/Robert\%20Pecho\%20 de\%20 la\%20Cruz,pdf): Acceso 12/01/2016.

Gentry, H. 1993. Woody Plants of Northwest South America (Colombia, Ecuador, Perú). With supplementary notes on Herbaceous Taxa. Conservation International. Washington, U.S.A. 879 pp.

Guevara, E. 2011. Propiedades físico-mecánicas de Heteropsis flexuosa "alambre tamshi", Thoracocarpus bissectus "cesto tamshi" y Desmoncus polyacanthos "cashavara", LoretoPerú. UNAP, Tesis de Ing. Forestal. 49 p más anexos.

Hoffman, B. 1997. The Biology and use of nibbi Heteropsis flexuosa (Araceae): the source of an aerial root fiber product in Guyana. MSc Thesis, Florida International University. Miami, USA. 147 pp.

IAWA (International Association of Wood Anatomists) Committee. 1989. List of Microscopic Features For Hardwood Identification. IAWA Bulletin, 10: 217-332 p.

IBAMA (Instituto Brasileiro Do Meio Ambiente E Dos Recursos Naturais Renováveis). 1991. Normas De Procedimientos Em Estudios Do Anatomía Do Madeira: I, Angiospermae.

JUNAC. 1989. Manual del grupo andino para el secado de maderas. Junta del Acuerdo de
Cartagena. Primera edición. Editorial Carbajal S. A. Colombia. 52 p.

Knab-Vispo, C; Hoffman, B; Moermond, T \& Vispo, C. 2000. Ecological Observations on Heteropsis spp. (Araceae) in southern Venezuela. The New York Botanical Garden. In: Economic Botany. Vol 57, N³.345-353 p. USA.

Saldaña, J. 2004. Biología, ecología y manejo de Heteropsis flexuosa (H.B.K.) Bunting alambre tamshi en Jenaro Herrera, Loreto-Perú. UNAP, Tesis de Ing. Forestal. 72 p. más anexos.

TAPPI (Technical Association of the Pulp and Paper Industry). 1967. T231 cm-84, Coarseness of pulp fibers. Tag and Label Manufacturers Institute (TLMI).

TAPPI (Technical Association of the Pulp and Paper Industry). 1996a. T494 om-96. Tensile properties of paper and paperboard.

TAPPI (Technical Association of the Pulp and Paper Industry). 1996b. T511 om-96. Folding endurance of paper (MIT tester). Tag and Label Manufacturers Institute (TLMI).

TAPPI (Technical Association of the Pulp and Paper Industry). 1997a. T411 om-97. Thickness (caliper) of paper, paperboard, and combined board. Tag and Label Manufacturers Institute (TLMI).

TAPPI (Technical Association of the Pulp and Paper Industry). 1997b. T412 om-94. Moisture in pulp paper and paperboard. Tag and Label Manufacturers Institute (TLMI).

TAPPI (Technical Association of the Pulp and Paper Industry). 1997c. T544 sp-97. Effect of moist heat on properties of paper and board. Tag and Label Manufacturers Institute (TLMI). Vásquez, R. 1997. Flórula de las Reservas Biológicas de Iquitos, Perú. Missouri Botanical Garden. V 63. USA. 1046 p.

Recibido: 28 de enero de 2019 Aceptado para publicación: 29 de abril de 2019 